\title{
Risk factors, hyposalivation and impact of xerostomia on oral health-related quality of life
}

\section{Sven NIKLANDER(a) Laura VEAS(a) \\ Cristina BARRERA(a) \\ Flavio FUENTES(a) \\ Giuliana CHIAPPINI(a) \\ Maureen MARSHALL(a)}

(a) Universidad Andres Bello, Dentistry Faculty, Viña del Mar, Chile.

Declaration of Interests: The authors certify that they have no commercial or associative interest that represents a conflict of interest in connection with the manuscript.

\section{Corresponding Author:}

Sven Niklander

E-mail: Sven.niklander@unab.cl

DOI: 10.1590/1807-3107BOR-2017.vol31.0014

Submitted: June 08, 2016

Accepted for publication: Nov 21, 2016

Last revision: Dec 06, 2016
Abstract: To determine xerostomia-related frequency, factors, salivary flow rates and Oral Health-Related Quality of Life (OHRQoL) of patients attending the Universidad Andrés Bello Dental School Clinic, in the city of Viña del Mar, Chile. The study involved 566 patients assessed with xerostomia, based on a single standardized questionnaire. The severity and impact of xerostomia on OHRQoL was assessed using a visual analogue scale (VAS) and the short version of the Oral Health Impact Profile Questionnaire (OHIP-14sp), respectively. Stimulated and non-stimulated salivary flow rates were obtained from a sample of patients. Xerostomia was reported in 61 patients (10.8\%), comprising 50 women $(83.3 \%)$ and 11 men $(16.7 \%)$ ( $p<0.013)$. The prevalence was $13 \%$ among the women and $6.1 \%$ among the men. Gender, age and medication were found to be independent risk factors for the development of xerostomia. Hyposalivation was found in 10 of the 35 patients with xerostomia (28.6\%) and in 2 patients without it $(\mathrm{p}<0.011)$. Patients with xerostomia had a reduced OHRQoL, compared with patients without xerostomia, as shown by the total OHIP-14sp score $(p<0.001)$. Xerostomia was a common, potentially debilitating condition with a major impact on the OHRQoL of a patient population attending a university-based dental clinic. Hyposalivation was present in almost $30 \%$ of the patients who complained of xerostomia. It is important that general dentists be aware of this condition, so that they can provide patients with a good diagnosis, treatment and follow-up.

Keywords: Xerostomia; Quality of Life.

\section{Introduction}

Saliva is one of the most essential fluids of the body. Patients never realize how important it is until it is missing, and ordinary things, like eating or speaking, become a daily problem. The sensation of dry mouth, or xerostomia, is a common complaint in clinical practice. Xerostomia is defined as "a subjective sensation of dry mouth," which is usually correlated with low salivary flow rates (hyposalivation). Nevertheless, many patients have a dry mouth sensation with normal salivary flow rates; therefore, xerostomia does not necessary reflect salivary gland hypofunction. Hyposalivation is defined as an objective reduction in the salivary flow rate. Saliva is usually categorized as unstimulated 
(resting) or stimulated. Unstimulated saliva reflects the basal flow rate aimed at protecting the oral mucosa, and has been reported to range from about $0.29 \mathrm{ml} / \mathrm{min}$ to $0.41 \mathrm{ml} / \mathrm{min}$. Stimulated saliva helps with mastication and digestion, and has been reported to be between $1-2 \mathrm{ml} / \mathrm{min}^{1}$

Xerostomia is a common problem. Its prevalence is difficult to determine, because it varies between different geographical zones and age groups, and because of differences in the inclusion criteria and methodology of studies. ${ }^{2}$ Epidemiological studies have reported its prevalence to be between $0.9 \%$ and $64.8 \%{ }^{3}$

Many factors have been associated with xerostomia. The most common causes are: $: 4,5,6,7,8,9$

a. Medications (more than 400 drugs are associated with xerostomia as a side effect);

b. Psychological conditions, such as stress and anxiety;

c. Salivary gland disorders, such as Sjögren's syndrome;

d. Head and neck radiotherapy.

Xerostomia is more common in women, and its prevalence is highest in the sixth decade, probably because of an increase in drug intake, due to chronic disorders, such as hypertension, diabetes, depression, etc. ${ }^{10,11}$ however, there are reports that age itself acts as a risk factor for xerostomia. Field et al. ${ }^{12}$ reported that the use of medications and age independently increases the likelihood of developing xerostomia 1.24 times for every 10 years of age. The same authors also reported almost four times greater prevalence of xerostomia in patients taking medications (28\%), compared with those not taking any (7.5\%). It has been reported that not only the type of drug, but also the number of drugs taken (polypharmacy, or simultaneous, multiple use of drugs) increases the likelihood of developing xerostomia, which is a common feature in elderly patients. ${ }^{13}$

Dry mouth sensation has many effects on oral health, and may have a negative impact on quality of life. Patients with xerostomia may complain of a burning sensation, abnormal taste, dysarthria, dysphagia, dysgeusia, halitosis and lack of retention of dentures. Examination may demonstrate oral dryness, thickening of saliva, evidence of candidal infection (usually angular cheilitis or erythematous candidiasis), tongue fissuring and depapillation, cervical caries and halitosis. ${ }^{14}$ It has been documented that xerostomia is a good predictor of root caries development in elderly patients. ${ }^{15}$

Little attention has been given to the impact of xerostomia on quality of life, in comparison with the effects of caries and periodontal disease. A study conducted in Canada, which evaluated oral health-related quality of life (OHRQoL) in patients with dry mouth, reported that xerostomia had an important influence on well-being and quality of life. Similar results have been published elsewhere. ${ }^{16,17}$

Although associated factors, salivary flow rates and prevalence of xerostomia have been reported in many studies, these features have strong geographical variations, and none of these studies were conducted among Chilean patients. Furthermore, OHRQoL in patients with xerostomia has not been widely studied, even though xerostomia is a common clinical condition with a growing prevalence.

The aim of this study was to determine the xerostomia-related frequency, factors, salivary flow rates and OHRQoL of patients attending a dental school clinic in the city of Viña del Mar, Chile. To our knowledge, this is the first study that has investigated the frequency, causes and impact on quality of life of xerostomia in a Chilean population.

\section{Methodology}

\section{Study population}

The sample size was calculated with a power of $90 \%$, a confidence level of $95 \%$, an estimated prevalence of $15 \%$ and an error of $5 \%$. The minimum number of required participants was 536 . The study involved 566 patients who attended the dental clinic of the Universidad Andrés Bello School of Dentistry in Viña del Mar, Chile, between April and November 2014. Approval was obtained from the scientific research ethics committee of the Universidad Andrés Bello School of Dentistry prior to the study. Participants in this study were recruited when they attended their dental appointment, and had to be over eighteen years old to be included. Every patient over eighteen attending the dental clinic during the study 
recruitment time was invited to participate. Eligible individuals who agreed to participate were required to sign a consent form before being interviewed. This research was conducted in full accordance with the World Medical Association Declaration of Helsinki. The data were collected by personal interview conducted at the dental clinic, and were recorded in a specifically designed questionnaire.

\section{Measurements}

\section{Xerostomia and related factors}

The questionnaire included the following variables: gender, age, systemic diseases, menopause, drugs (type, number and time of use), smoking habits (measured as number of cigarettes consumed per day) and presence of xerostomia.

The following question was asked to evaluate the presence of xerostomia: "How often do you feel that your mouth is dry?" Participants could select from the following answers: 'never,' 'sometimes,' 'usually' or 'always.' Those who answered 'usually' or 'always' were considered as suffering from xerostomia. 18,19,20

The severity of xerostomia was measured using a visual analogue scale (VAS). The visual analogue scale consisted of a $10-\mathrm{cm}$ horizontal line with 0 and 10 marked on each extreme. Zero reflected no xerostomia and ten, the worst degree of xerostomia. Patients were asked to draw a vertical line perpendicular to this horizontal line to reflect their symptom severity. The distance from the vertical line to zero was measured and recorded to determine the value for each patient. . $^{21,22}$

\section{Evaluation of xerostomia impact on quality of life}

Oral health-related quality of life was assessed using the Spanish version of the oral health impact profile-14 questionnaire (OHIP-14sp). OHIP-14 is a 14-item questionnaire designed to measure self-reported functional limitation, discomfort and disability attributed to oral conditions. ${ }^{23}$ Although the OHIP-14sp is a short questionnaire, it has been found to be reliable and sensitive to changes, and to have adequate cross-cultural consistency. ${ }^{24} \mathrm{OHIP}-14 \mathrm{sp}$ was evaluated according to the following domains: functional limitation, physical pain, psychological discomfort, physical incapacity, psychological incapacity, social incapacity, and social disadvantage. The answers were assessed using a Likert type evaluation scale with five points: never $=0$; rarely $=1$; sometimes $=2$; repeatedly $=3$; always $=4$. The OHIP-14sp scale ranges from 0 to 56 . The lowest scores represent a satisfactory perception of an individual's oral conditions, and, therefore, higher satisfaction and better quality of life.

\section{Evaluation of salivary flow rate}

A case control study was conducted to correlate the presence of xerostomia and salivary flow rate. All the 61 patients who complained of xerostomia were invited to participate in this part of the study. However, patients who had any disease associated with xerostomia (such as Sjögren's Syndrome, hepatitis C, HIV, etc.) were excluded. Thirty-five patients who had xerostomia and met the inclusion criteria accepted to participate in this part of the study, and were assigned to the xerostomia group. Thirty-five patients without xerostomia were invited to compose the control group. These were selected with a simple random sampling method using Microsoft Excel 2011, among the patients who answered 'never' to the xerostomia question. Two investigators were responsible for selecting these patients, and they were not blinded to the diagnosis. Additionally, these patients were asked to answer five questions about presence or absence of oral symptoms (eating impairment, burning sensation, abnormal taste, self-perceived halitosis and need to drink liquids to aid in swallowing foods).

Sialometry was performed using the spitting method. Patients were instructed to refrain from eating, drinking, smoking and engaging in oral hygiene activities for a minimum of $60 \mathrm{~min}$ before the procedure. Samples were collected in the morning hours, between 9:30 and 11:30 am, always in the same room under similar room temperatures. Collection time for stimulated and non-stimulated whole salivary flow was 5 minutes. First, non-stimulated whole saliva was collected. Patients were instructed to spit into a tube for five minutes, and the amount of saliva was measured using a graduated syringe. Stimulated whole saliva was collected after an interval of 3 minutes using the mastication method. Subjects were asked to 
chew a wax cube $15 \mathrm{~mm} \times 10 \mathrm{~mm}$ for 1 minute at their own pace, and then to spit into a tube for 5 minutes. Wax residue was eliminated using filter paper before quantification using a graduated syringe. The cut-off values for hyposalivation were based on previous literature, and were as following: below $0.1 \mathrm{ml} / \mathrm{min}$ for unstimulated whole salivary flow and below $0.5 \mathrm{ml} / \mathrm{min}$ for stimulated whole salivary flow. ${ }^{1}$ The Lin's concordance agreement between the examiners was 0.9 .

\section{Statistical analysis}

The data was analyzed using Microsoft Excel 2011, R-Cran 3.0.1 (version 64x) and IBM SPSS Statistics, version 22 for Windows.

Xerostomia analysis: The Fisher exact test and the Wilcoxon rank sum test were used to analyze qualitative and quantitative variables, respectively. Logistic regression was used to determine associated factors.

Salivary flow rates, hyposalivation and oral symptoms analysis: The Wilcoxon rank sum test was applied for quantitative variables, and the chi-square test was used for qualitative variables.

P-values below 0.05 were considered statistically significant.

\section{Results}

The study population comprised 566 participants ( 386 women and 180 men) aged 18 to 83 , with a mean age of 46 years (SD 15.59).
Xerostomia was reported by 61 patients (10.8\%). Of these, 50 were women $(83.3 \%$ ) and 11 were men $(16.7 \%)$ $(\mathrm{p}<0.013)$. The prevalence of xerostomia was $13 \%$ among the women [CI 95\%: 9.8\%-16.6\%] and 6.1\% among the men [CI 95\%: 3.1\%-10.7\%]. The highest prevalence was seen in patients aged 68 to 77 years (33.3\%), followed by patients aged 78 to $83(22.27 \%)$ (Table 1$)$.

A total of 372 patients of the sample population had a systemic disease, and 194 were systemically well. Fifty patients with xerostomia (82\%) had a systemic disease, and 11 (18\%) were systemically well. Cardiovascular diseases, psychological illness, gastrointestinal diseases and allergies were the most commonly reported diseases. Nevertheless, there was no statistical association between the presence of a systemic disease and xerostomia ( $p>0.442$ ). The presence of menopause was not statistically associated with xerostomia ( $p=0.052)$. Nevertheless, a higher prevalence of xerostomia was found among menopausal women (17.97\% vs $10.47 \%)$.

Of the 566 patients, $42.4 \%(n=240)$ reported taking some type of medication. The prevalence of xerostomia in these patients was $17.92 \%$. The prevalence of xerostomia among the patients not taking medication was $5.52 \%$. This difference was statistically significant ( $\mathrm{p}<0.001)$. Of the 61 patients with xerostomia, $70.5 \%$ $(n=43)$ were taking one or more medications at the time of the study. A statistically significant association was found between the use of antidepressants $(p<0.001)$, antihypertensives $(p<0.001)$ and antihistamines $(p=0.045)$ with xerostomia.

Table 1. Prevalence of xerostomia by age group.

\begin{tabular}{|c|c|c|c|c|c|c|}
\hline \multirow{3}{*}{ Age } & \multicolumn{4}{|c|}{ Presence of xerostomia } & \multirow{2}{*}{\multicolumn{2}{|c|}{ Total }} \\
\hline & \multicolumn{2}{|c|}{ Yes } & \multicolumn{2}{|c|}{ No } & & \\
\hline & no. & $\%$ & no. & $\%$ & no. & $\%$ \\
\hline $18-27$ & 6 & 5.94 & 95 & 94.06 & 101 & 100.00 \\
\hline $28-37$ & 7 & 7.87 & 82 & 92.13 & 89 & 100.00 \\
\hline $38-47$ & 12 & 10.62 & 101 & 89.38 & 113 & 100.00 \\
\hline $48-57$ & 15 & 10.87 & 123 & 89.13 & 138 & 100.00 \\
\hline $58-67$ & 7 & 8.64 & 74 & 91.36 & 81 & 100.00 \\
\hline $68-77$ & 11 & 33.33 & 22 & 66.67 & 33 & 100.00 \\
\hline $78-87$ & 3 & 27.27 & 8 & 72.73 & 11 & 100.00 \\
\hline Total & 61 & 10.78 & 505 & 89.22 & 566 & 100.00 \\
\hline
\end{tabular}




\section{Associated factors for xerostomia}

Age alone was found to be a factor associated with xerostomia. For every one-year increment, the chances of having xerostomia increased 1.01 times $(\mathrm{p}<0.001)$. Males were found to have a 1.56 times smaller chance of having xerostomia, compared with females ( $p<0.012)$. The chance of having xerostomia increased 1.12 times with each additional medication being taken $(p=0.005)$. Systemic diseases and smoking habit were not found to be associated factors in xerostomia development (Table2).

\section{Oral health-related quality of life}

As shown in Table 3, patients with xerostomia had a significantly higher total OHIP-14sp score than patients without xerostomia (20.1 \pm 14.32 and $12.7 \pm 12.9$ respectively, $\mathrm{p}<0.001)$. Patients with xerostomia scored higher on every domain of the OHIP-14sp ( $p<0.005)$ than patients without it, with a higher impact on psychological discomfort, psychological incapacity and physical pain $(4.1,3.8$ and 3.4, respectively) (Table 4).

Table 2. Logistic regression for associated factor analysis.

\begin{tabular}{lccccc}
\hline Variables & $\begin{array}{c}\text { Degrees of } \\
\text { freedom }\end{array}$ & Deviance & $\begin{array}{c}\text { Residual degrees } \\
\text { of freedom }\end{array}$ & Residual deviance & $p$-value \\
\hline Age & 1 & 10.123 & 564 & 376.83 & 0.001464 \\
Gender & 1 & 6.189 & 563 & 370.65 & 0.012853 \\
Presence of systemic disease & 1 & 0.591 & 562 & 370.05 & 0.442083 \\
$\mathrm{~N}^{\circ}$. of medications & 1 & 7.749 & 561 & 362.31 & 0.005373 \\
$\mathrm{~N}^{\circ}$ of cigarettes & 1 & 0.892 & 560 & 361.41 & 0.344942 \\
\hline
\end{tabular}

Table 3. Patient-reported symptoms.

\begin{tabular}{|c|c|c|c|}
\hline \multirow{2}{*}{ Variable } & \multicolumn{2}{|c|}{ Frequency n(\%) } & \multirow{2}{*}{$\mathrm{p}$-value } \\
\hline & Xerostomia & Control & \\
\hline Eating impairment & $7(20)$ & $0(0)$ & $<0.011$ \\
\hline Need to drink liquids to aid in swallowing foods & $9(25)$ & $0(0)$ & $>0.057$ \\
\hline Burning sensation & $7(20)$ & $1(2.9)$ & $>0.055$ \\
\hline Abnormal taste & $12(34.3)$ & $3(8.6)$ & $<0.009$ \\
\hline Self-perceived halitosis & $14(40)$ & $8(22.9)$ & $>0.122$ \\
\hline
\end{tabular}

Table 4. Comparison of the OHIP-14sp scores for xerostomia vs. non-xerostomia patients.

\begin{tabular}{|c|c|c|c|c|c|c|}
\hline \multirow{2}{*}{ OHIP domains } & & \multicolumn{2}{|c|}{ With xerostomia } & \multicolumn{2}{|c|}{ Without xerostomia } & \multirow{2}{*}{$\mathrm{p}$-value } \\
\hline & & Mean & Standard deviation & Mean & Standard deviation & \\
\hline Dom. 1 & Functional limitation & 2.03 & 1.59 & 0.97 & 1.4 & $<0.001$ \\
\hline Dom.2 & Physical pain & 3.43 & 2.01 & 2.3 & 1.87 & $<0.001$ \\
\hline Dom.3 & Psychological discomfort & 4.15 & 2.12 & 3.28 & 2.07 & $<0.004$ \\
\hline Dom.4 & Physical incapacity & 2.51 & 2.09 & 1.42 & 1.87 & $<0.001$ \\
\hline Dom.5 & Psychological incapacity & 3.80 & 2.18 & 2.33 & 2.16 & $<0.001$ \\
\hline Dom.6 & Social incapacity & 2.13 & 2.06 & 1.26 & 1.84 & $<0.001$ \\
\hline Dom.7 & Social disadvantage & 2 & 2.27 & 1.15 & 1.78 & $<0.001$ \\
\hline Total OHIP & & 20.05 & 14.32 & 12.71 & 12.99 & - \\
\hline
\end{tabular}


Cronbach's alpha coefficient was 0.879 for the OHIP-14, considered a good internal consistency reliability score. ${ }^{25}$

\section{Salivary flow rates / hyposalivation}

Seventy patients ( 35 with and 35 without xerostomia) were divided into two groups: a xerostomia and a control group ( 35 patients in each group). The xerostomia group consisted of 29 females and 6 males, with a mean age of $53.8 \pm 16.6$ years. The control group consisted of 24 females and 11 males with a mean age of $46.6 \pm 14.6$.

Mean non-stimulated and stimulated whole salivary flow was $0.1 \pm 0.28 \mathrm{ml} / \mathrm{min}$ and $1.53 \pm 1.03 \mathrm{ml} / \mathrm{min}$ for the xerostomia group, respectively, and $0.42 \pm 0.33 \mathrm{ml} / \mathrm{min}$ and $1.53 \pm 0.96 \mathrm{ml} / \mathrm{min}$ for the control group, respectively. No statistically significant differences were found between the groups, neither for non-stimulated nor for stimulated whole salivary flow rates.

Non-stimulated hyposalivation (defined above as a salivary flow rate below $0.1 \mathrm{ml} / \mathrm{min}$ ) was found in $28.6 \%$ $(\mathrm{n}=10)$ of the xerostomia group patients, and in 5.7\% $(n=2)$ of the control group patients. This difference was statistically significant $(p<0.011)$. Stimulated hyposalivation (defined above as a salivary flow rate below $0.5 \mathrm{ml} / \mathrm{min}$ ) was found in $14.3 \%(\mathrm{n}=5)$ of the xerostomia group patients, and in $8.6 \%(n=3)$ of the control group patients. These values were not statistically significant $(p>0.452)$.

\section{Oral symptoms}

Eating impairment and abnormal taste sensation were more frequent among patients with xerostomia, with statistically significant differences $(\mathrm{p}<0.01$ and $p<0.009$, respectively). Although burning sensation, self-perceived halitosis and the need to drink liquids to aid in swallowing foods, were more common among the xerostomia group, the value was not statistically significant (Table 3).

\section{Discussion}

The prevalence of xerostomia in a Chilean population attending a dental school clinic in Viña del Mar, Chile, was $10.8 \%$. Orellana et al. conducted a systematic review in which they reported a prevalence of xerostomia between $0.9 \%$ and $64.8 \%$, but most of the reports involved people over 50 years of age. ${ }^{3}$
In accordance with the present results, Hahnel et al. ${ }^{25}$ reported a prevalence of $16 \%$, and Field et al., ${ }^{12}$ one of $12.7 \%$, in a general population. In a Swedish study, the authors reported a $22 \%$ prevalence of xerostomia in patients aged 20 to 60 years. ${ }^{27}$ Nederfors et al. estimated the prevalence in the Swedish population to be $21.3 \%$ for men and $27.3 \%$ for women. ${ }^{28}$ Österberg et al. ${ }^{29}$ and Locker ${ }^{30}$ reported prevalence rates of $21 \%$ and $22.5 \%$ for men and women over 60 years, respectively.

Because the dry mouth sensation is a subjective symptom, it is difficult to compare prevalence rates among studies, due to the different methods of assessment. The fact that there is no "single universal question" that could be asked to assess xerostomia makes comparisons among different studies difficult. It is important when comparing prevalence rates among studies to consider the age range of the study population. Most of the studies that report prevalence rates higher than the present results were carried out in groups of older patient (over 50 or over 60 years of age), therefore correlating with more risk factors, and possibly explaining the higher prevalence rates. Nevertheless, these studies do not afford precise information on how common this problem is in the general population.

It is widely reported that xerostomia is more common among women. ${ }^{3,28}$ Many have argued that this may be because women take more medications than men, but it has been found that even non-medicated women have a higher prevalence of xerostomia than men. ${ }^{2}$ A recent systematic review ${ }^{3}$ indicated that the prevalence of xerostomia in females is $8 \%$ higher than in males. The results of the present study corroborate this, showing a difference of $7 \%$ between the genders (13\% in women and $6.1 \%$ in men). Based on the present results, men have a 1.56 times smaller chance of presenting with xerostomia than women. Similarly, Thomson et al. reported xerostomia to be 1.62 times more likely to occur in women than in men. ${ }^{31}$

Several studies have reported xerostomia to be more frequently related with older age, especially in populations over 60 years of age..$^{2,26}$ The explanation may be that age acts as a risk factor, or it could be that elderly patients have more risk factors than younger people. Older age is associated with more chronic diseases, which is associated with more drug intake, and this is the most common cause of dry mouth. 
It has been reported that age per se could act as a risk factor for the development of dry mouth, since old people may have diminished salivary gland secretion, due to salivary gland atrophy. ${ }^{28}$ An English study studied over 1,100 patients to determine risk factors for xerostomia. The authors concluded that age in itself increases the risk of having dry mouth 1.24 times for every 10 years of age. ${ }^{12}$ The present study also found age to be a risk factor for xerostomia. Every additional year of life increases the chances of having dry mouth 1.01 times.

Although age in itself can be considered a risk factor, a better predictor of the development of xerostomia is the use of medication. Based on the results of the present study, dry mouth sensation is more common in medicated patients ( $17.92 \%$ vs. $5.52 \%)$, and the likelihood of having xerostomia increases 1.12 times for each additional medication taken. Han et al. reported a $33.5 \%$ prevalence of xerostomia in patients with a daily intake of at least three drugs, and a $67 \%$ prevalence in patients who take more than seven drugs a day. ${ }^{2}$ Yuan et al. found an increase in the prevalence of dry mouth when patients took more than three medications. ${ }^{32}$ Field et al. ${ }^{12}$ concluded that medication is a highly significant risk factor for having a dry mouth, and is a better predictor of risk status than either age or gender. This corroborates the study by Nederfors et al., ${ }^{28}$ who found a very strong association between polypharmacy and xerostomia.

Corroborating the results of this report, a recent systematic review on medication-induced salivary gland dysfunction concluded that xerostomia is more common among medicated persons than among non-medicated persons and that the frequency of xerostomia is mostly related to the dose and number of medications. ${ }^{6}$

It is reported that menopause may act as a possible risk factor for reporting a dry mouth. ${ }^{10,33}$ Although the present report found a dry mouth prevalence of $17.97 \%$ among menopausal women, this was not statistically significant when compared with non-menopausal women $(p=0.052)$.

Patients with xerostomia have a more reduced OHRQoL than patients without xerostomia, as shown in the total OHIP-14sp score. The highest impact was detected for the domains of psychological discomfort, psychological incapacity and physical pain. Nevertheless, the utility of the OHIP-14sp in detecting changes in the different sub-scales has been questioned; ${ }^{34}$ thus, this result should be regarded with caution. According to Hopcraft et al., patients with severe xerostomia are 2.3 to 4.9 times more likely to have a negative experience and negative impact on quality of life. ${ }^{35}$ A Japanese study concluded that xerostomia is independently associated with a higher impact on OHRQoL. ${ }^{36}$ According to some authors, xerostomia affects quality of life by interfering with speech, taste and mood. ${ }^{17}$ Patients with xerostomia are more prone to having dental caries, periodontal disease, burning sensation and lack of retention of dentures, which are also related with a reduced OHRQoL. ${ }^{35}$ Hanel et al. ${ }^{26}$ reported a positive correlation between the severity of xerostomia and impact on OHRQoL - more severe symptoms are associated with a higher impact on OHRQoL.

Although there were no significant differences between the non-stimulated and the stimulated whole salivary flow rates (measured in $\mathrm{ml} / \mathrm{min}$ ) between patients with and without xerostomia, there was a significant association between non-stimulated hyposalivation and xerostomia $(p<0.01)$. This is important in clinical practice; the present results indicate that almost $30 \%$ of patients who complain of xerostomia of non-organic cause will have a non-stimulated salivary flow rate below $0.1 \mathrm{ml} / \mathrm{min}$, which could have a detrimental effect on the oral cavity.

Eating impairment and abnormal taste sensation were associated with xerostomia, as corroborated by Lee et al. ${ }^{37}$ Although burning sensation and the need to drink liquids to aid in swallowing foods were not statistically associated with xerostomia in this study, the $\mathrm{p}$ values were close to significance. The relation between xerostomia and the need to drink liquids to aid in swallowing foods was not found to be statistically significant. It is noteworthy that this concept is used in many studies as a single question to diagnose xerostomia. Nonetheless, study results suggest that this question should no longer be used to diagnose xerostomia, since it is not strongly associated with xerostomia, if at all. A larger sample size is needed to clarify this. 
This study has two limitations. First, our population was recruited from a university dental clinic, suggesting that the prevalence of xerostomia might be overestimated. Therefore, more population-based studies are warranted. Second, we recognize that it would have been ideal to assess xerostomia using a specially designed questionnaire, such as the xerostomia inventory, but the validated Spanish version of this questionnaire was not available when this study was conducted.

\section{Conclusion}

Dry mouth sensation is a common problem among Chilean patients, with a prevalence of $10.8 \%$. Risk factors for dry mouth are gender, age, and, most importantly, the type and number of medications taken. Non-stimulated hyposalivation may be present in about $30 \%$ of patients with a non-organic cause of xerostomia. Dry mouth sensation is a potentially debilitating condition that can considerably affect the OHRQoL. It is important that general dentists be aware of this condition and understand its possible causes and risk factors, so that they can provide patients with a good diagnosis, treatment and follow-up.

\section{Acknowledgements}

We greatly appreciate the grammatical correction of the manuscript by Michael Freedman. This research received financial support from Universidad Andrés Bello, grant: UNABDI-822-15/CB.

\section{References}

1. Sreebny LM. Saliva in health and disease: an appraisal and update. Int Dent J. 2000;50(3):140-61. doi:10.1111/j.1875-595X.2000.tb00554.x

2. Han P, Suarez-Durall P, Mulligan R. Dry mouth: a critical topic for older adult patients. J Prosthodont Res. 2015;59(1):6-19. doi:10.1016/j.jpor.2014.11.001

3. Orellana MF, Lagravère MO, Boychuk DG, Major PW, FloresMir C, Ortho C. Prevalence of xerostomia in populationbased samples: a systematic review. J Public Health Dent. 2006;66(2):152-8. doi:10.1111/j.1752-7325.2006.tb02572.x

4. González S, Sung H, Sepúlveda D, González M, Molina C. Oral manifestations and their treatment in Sjogren's syndrome. Oral Dis. 2014;20(2):153-61. doi:10.1111/odi.12105

5. Ohara Y, Hirano H, Yoshida H, Obuchi S, Ihara K, Fujiwara Y, et al. Prevalence and factors associated with xerostomia and hyposalivation among community-dwelling older people in Japan. Gerodontology. 2016;33(1):20-7. doi:10.1111/ger.12101

6. Aliko A, Wolff A, Dawes C, Aframian D, Proctor G, Ekström J et al. World Workshop on Oral Medicine VI: clinical implications of medication-induced salivary gland dysfunction. Oral Surg, Oral Med Oral Pathol Oral Radiol. 2015;120(2):185-206. doi:10.1016/j.oooo.2014.10.027

7. Saleh J, Figueiredo MA, Cherubini K, Salum FG. Salivary hypofunction: an update on aetiology, diagnosis and therapeutics. Arch Oral Biol. 2015;60(2):242-55. doi:10.1016/j.archoralbio.2014.10.004

8. Schubert MM, Izutsu KT. Iatrogenic causes of salivary gland dysfunction. J Dent Res. 1987;66(spec no):680-8. doi:10.1177/00220345870660S213

9. Scully C. Drug effects on salivary glands: dry mouth. Oral Dis. 2003;9(4):165-76. doi:10.1034/j.1601-0825.2003.03967.x
10. Ship JA. Diagnosing, managing, and preventing salivary gland disorders. Oral Dis. 2002;8(2):77-89. doi:10.1034/j.1601-0825.2002.2o837.x

11. Scully C, Bagan JV. Adverse drug reactions in the orofacial region. Crit Rev Oral Biol Med. 2004;15(4):221-39. doi:10.1177/154411130401500405

12. Field EA, Fear S, Higham SM, Ireland RS, Rostron J, Willetts RM, et al. Age and medication are significant risk factors for xerostomia in an English population, attending general dental practice. Gerodontology. 2001;18(1):21-4 . doi:10.1111/j.1741-2358.2001.00021.x

13. Villa A, Abati S. Risk factors and symptoms associated with xerostomia: a cross-sectional study. Aust Dent J. 2011;56(3):290-5. doi:10.1111/j.1834-7819.2011.01347.x

14. Wolff A, Fox PC, Ship JA, Atkinson JC, Macynski AA, Baum BJ. Oral mucosal status and major salivary gland function. Oral Surg Oral Med Oral Pathol. 1990;70(1):49-54. doi:10.1016/0030-4220(90)90177-T

15. Kitamura M, Kiyak HA, Mulligan K. Predictors of root caries in the elderly. Community Dent Oral Epidemiol.1986;14(1):34-8. doi:10.1111/j.1600-0528.1986.tb01491.x

16. Gerdin EW, Einarson S, Jonsson M, Aronsson K, Johansson I. Impact of dry mouth conditions on oral health-related quality of life in older people. Gerodontology 2005;22(4):219-26. doi:10.1111/j.1741-2358.2005.00087.x

17. Willumsen T, Fjaera B, Eide H. Oral health-related quality of life in patients receiving home-care nursing: associations with aspects of dental status and xerostomia. Gerodontology 2010;27(4):251-7. doi:10.1111/j.1741-2358.2009.00344.x 
18. Murray Thomson W, Poulton R, Mark Broadbent J, Al-Kubaisy S. Xerostomia and medications among 32-year-olds. Acta Odontol Scand. 2006;64(4):249-54. doi:10.1080/00016350600633243

19. Thomson WM, Brown RH, Williams SM. Medication and perception of dry mouth in a population of institutionalised elderly people. N Z Med J. 1993;106(957): 219-21.

20. Gómez-Moreno G, Guardia J, Aguilar-Salvatierra A, Cabrera-Ayala M, Maté-Sánchez de-Val JE, Calvo-Guirado JL. Effectiveness of malic acid $1 \%$ in patients with xerostomia induced by antihypertensive drugs. Med Oral Patol Oral Cir Bucal. 2013;18(1): e49-e55. doi:10.4317/medoral.18206

21. Furness S, Worthington HV, Bryan G, Birchenough S, McMillan R. Interventions for the management of dry mouth: topical therapies. Cochrane Database Syst Rev. 2013;9(12):CD008934. doi:10.1002/14651858.CD008934.pub2

22. Davies AN, Daniels C, Pugh R, Sharma K. A comparison of artificial saliva and pilocarpine in the management of xerostomia in patients with advanced cancer. Palliat Med. 1998;12(2):105-11. doi:10.1191/026921698670435903

23. Ni Riordain R, McCreary C. The use of quality of life measures in oral medicine: a review of the literature. Oral Dis. 2010;16(5):419-30. doi:10.1111/j.1601-0825.2009.01647.x

24. Montero-Martín J, Bravo-Pérez M, Albaladejo-Martínez A, Hernández-Martín LA, Rosel-Gallardo EM. Validation the Oral Health Impact Profile (OHIP-14sp) for adults in Spain. Med Oral Patol Oral Cir Bucal. 2009;14(1):44-50.

25. Ni Riordain R, McCreary C. Validity and reliability of a newly developed quality of life questionnaire for patients with chronic oral mucosal diseases. J Oral Pathol Med. 2011;40(8):604-9. doi:10.1111/j.1600-0714.2011.01021.x

26. Hahnel S, Schwarz S, Zeman F, Schäfer L, Behr M. Prevalence of xerostomia and hyposalivation and their association with quality of life in elderly patients in dependence on dental status and prosthetic rehabilitation: a pilot study. J Dent. 2014;42(6):664-70. doi:10.1016/j.jdent.2014.03.003

27. Flink H, Bergdahl M, Tegelberg A, Rosenblad A, Lagerlof F. Prevalence of hyposalivation in relation to general health, body mass index and remaining teeth in different age groups of adults. Community Dent Oral Epidemiol. 2008;36(6):523-31. doi:10.1111/j.1600-0528.2008.00432.x
28. Nederfors T, Isaksson R, Mörnstad H, Dahlöf C. Prevalence of perceived symptoms of dry mouth in an adult Swedish population: relation to age, sex and pharmacotherapy. Community Dent Oral Epidemiol. 1997;25(3):211-6. doi:10.1111/j.1600-0528.1997.tb00928.x

29. Österberg T, Landahl S, Hedegård B. Salivary flow, saliva, $\mathrm{pH}$ and buffering capacity in 70-year-old men and women. Correlation to dental health, dryness in the mouth, disease and drug treatment. J Oral Rehabil.1984;11(2):157-70. doi:10.1111/j.1365-2842.1984.tb00565.x

30. Locker D. Xerostomia in older adults: a longitudinal study. Gerodontology. 1995;12(1):18-25. doi:10.1111/j.1741-2358.1995.tb00125.x

31. Murray Thomson W, Chalmers JM, John Spencer A, Slade GD, Carter KD. A longitudinal study of medication exposure and xerostomia among older people. Gerodontology. 2006;23(4):205-13. doi:10.1111/j.1741-2358.2006.00135.x

32. Yuan A, Woo SB. Adverse drug events in the oral cavity. Oral Surg Oral Med Oral Pathol Oral Radiol. 2015;119(1):35-47. doi:10.1016/j.oooo.2014.09.009

33. Santosh P, Nidhi S, Sumita K, Farzan R, Bharati D, Ashok K. Oral findings in postmenopausal women attending dental hospital in Western part of India. J Clin Exp Dent.2013;5(1):e8-12. doi:10.4317/jced.50928

34. Santos CM, Oliveira BH, Nadanovsky P, Hilgert JB, Celeste RK, Hugo FN. The Oral Health Impact Profile-14: a unidimensional scale? Cad Saúde Pública. 2013;29(4):749-57. doi:10.1590/S0102-311X2013000800012

35. Hopcraft MS, Tan C. Xerostomia: an update for clinicians. Aust Dent J. 2010;55(3):238-44. doi:10.1111/j.1834-7819.2010.01229.x

36. Ikebe K, Matsuda K, Morii K, Wada M, Hazeyama T, Nokubi $\mathrm{T}$ et al. Impact of dry mouth and hyposalivation on oral health-related quality of life of elderly Japanese. Oral Surg Oral Med Oral Pathol Oral Radiol Endod. 2007;103(2):216-22. doi:10.1016/j.tripleo.2005.12.001

37. Lee E, Lee YH, Kim W, Kho HS. Self-reported prevalence and severity of xerostomia and its related conditions in individuals attending hospital for general health examinations. Int J Oral Maxillofac Surg. 2014;43(4):498-505. doi:10.1016/j.ijom.2013.10.011 